\title{
Method of assigning spreading factor to improve the scalability of the LoRaWAN wide area network
}

\author{
Aleksandra Tiurlikova \\ National Research University \\ Higher School of Economics \\ Moscow, Russia \\ tyrlikova@mail.ru
}

\author{
Nikita Stepanov \\ Saint Petersburg State University \\ of Aerospace Instrumentation \\ Saint Petersburg, Russia \\ nstepanov@k36.org
}

\author{
Konstantin Mikhaylov \\ Centre for Wireless Communications \\ University of Oulu \\ Oulu, Finland \\ konstantin.mikhaylov@oulu.fi
}

\begin{abstract}
In modern world Low Power Wide Area Network (LPWAN) technologies have become the key enabler for a sheer diversity of the Internet of Things (IoT) applications involving massive deployment of resource-limited devices. In this paper we address the problem of improving the network scalability for one of the most widely used today licensee-free-band LPWAN technologies, named LoRaWAN. We show that the conventional method for assigning the spreading factor (SF) parameter to the devices in a LoRaWAN network, which effectively minimizes the consumption of individual devices, has some drawbacks when this comes to the scalability of the network as whole. Therefore, in this paper we propose another method of assigning the SFs to the nodes, which improves the probability of data delivery in an LPWAN at a cost of minor increase of the devices' consumption. The results of the conducted simulations confirm and characterize the utility of the proposed method.

Index Terms-LoRa; $L P W A N$; long range; low power; wide area network; energy consumption $I o T$; wireless; communication; scalability.
\end{abstract}

\section{INTRODUCTION}

The Internet of Things (IoT) is booming today and the range of its use cases is really tremendous. Among the whole sheer diversity of the technologies providing connectivity for the IoT (refer, e.g., to [1]), the ones, which represent the group of Low Power Wide Area Network (LPWAN) [2], are close to become the major driver for the applications implying massive deployment of devices with limited connectivity requirements. In this respect the LPWAN technologies reside between the existing cellular mobile network technologies and the short-range technologies, providing a cost and energy efficient connectivity solution for such devices as smart meters, infrastructure and environmental sensors, or tracking devices.

In order to reduce both capital (CAPEX) and operational expenditures (OPEX), the LPWAN technologies utilize several specific techniques. First, they often operate in license-free Industrial Scientific and Medical (ISM) frequency bands (either sub-GHZ: e.g., $868 \mathrm{MHz}$ for Europe or $915 \mathrm{MHz}$ for USA, or $2.4 \mathrm{GHz}$ band which is uniform worldwide) in order to avoid payment of spectrum license fees. Second, the network is built in star-of-starts manner, having a network server (NS) in the core, the gateways (GWs) around it and the end devices (EDs), communicating with the NS through the GWs. In order to keep the expenditures low, the GWs can be located far away from each other, resulting in the requirement of supporting a radio channel budget between an ED and a GW of over $130 \mathrm{~dB}$. To achieve this, the LPWAN EDs can operate with very low practical data rates - in the order of hundred bits per second. At the very same time, in order to ensure low expenditures the LPWAN technologies are often based on rather simple modulation-coding schemes and media access protocols, thus enabling for cost and energy efficient implementation of ED chipsets. Given this, and the fact that the LPWA technologies are primarily intended for the massive deployments, the good scalability is treated as one of the key performance indicators and the most critical challenge for these technologies [2], [3].

The recent market analyses show that among dozens of the LPWAN and LPWAN-like technologies available today, the one named LoRaWAN is among the most popular ones [4]. In the essence, the LoRaWAN technology combines the proprietary LoRa modulation developed by Semtech company, and an open-source media access (MAC) and network level (NWK) standard developed by the LoRa Aliiance. One of the key features of this technology is the use of chirpspread-spectrum modulation technique with variable spreading factor (SF), appended on top of the linearly changing carrier frequency. The SFs used by an ED can be changed thus enabling adaptation to the radio channel conditions by trading the on-air transmission time for the communication range. The spreading sequences for the different SFs are selected in a way to produce orthogonal radio signals, thus minimizing the interference between transmissions from the different EDs. To give a practical example, the SFs possible for a typical LoRaWAN network operating in EU $868 \mathrm{MHz}$ ISM band are listed in Table I. Note, that the LoRaWAN specification [5] defines several MAC options, called classes, with class A being the basic and the most commonly used one. In the essence, the operation of a class A ED is very close to that of the classical ALOHA, extended with frequency hopping and the two downlink slots added after each uplink transmission.

The LoRaWAN specification itself [5], and the only one known to us earlier study addressing this problem (i.e., [7]), suggest an ED to select the minimum SF possible. This is done in order to minimize the time spent by an ED for transmitting an uplink (and receiving the downlink) thus increasing the ED's energy utility. Even though this "mintime" strategy makes perfect sense for the sparse networks 
TABLE I

Modulation-CODing Schemes (I.E., DATA RATEs (DRs)) FOR LoRAWAN FOR 868 MHz EU BAND [5], [6]

\begin{tabular}{|c|c|c|c|c|c|}
\hline DR & Modulation & SF & Bandwidth, kHz & Max. payload, bytes & On-air time for 8-byte packet, ms \\
\hline 0 & LoRa & 12 & 125 & 59 & 59 \\
1 & LoRa & 11 & 125 & 59 & 790.528 \\
2 & LoRa & 10 & 125 & 123 & 252.608 \\
3 & LoRa & 9 & 125 & 230 & 127.9 \\
4 & LoRa & 7 & 125 & 230 & 70.91 \\
5 & LoRa & 7 & 250 & 230 & 35.46 \\
$6^{\text {a }}$ & LoRa & n/a & 50 & 230 & 5 \\
$7^{\text {a }}$ & FSK & & & \\
\hline
\end{tabular}

of infrequently transmitting devices, in the case of denselypopulated networks it may have some drawbacks. Therefore, in this paper we propose another method for assigning the SF for the EDs in a LoRaWAN network, which outperforms the "min-time" strategy in the probability of data delivery. By simulating the operation of a network of LoRaWAN devices for several illustrative set ups, we characterize the performance of the proposed method and compare it against to that for the state-of-the-art (SotA) "min-time" strategy. The proposed SF assignment method and the results of it's evaluation form the major contribution of this paper.

\section{Proposed SF ASSIGNMENT StRATEGY}

\section{A. System model}

Let us consider a city district. A single LoRaWAN GW is located in the middle of the circle of a specific radius. In average, every ten minutes an ED sends an 8-byte packet of data to the GW in uplink with no acknowledgments. Note, that we assume that the interval between the packets is exponentially distributed with parameter $\lambda$, and that no downlink communication is present. The transmit power of the EDs is constant (e.g., $14 \mathrm{dBm}$ ). For the sake of simplicity in what follows we consider only a single $125 \mathrm{kHz}$ broad channel with a carrier frequency of $868.100 \mathrm{MHz}$ with no duty cycle (DC) restrictions. The results for multiple frequency channels can be obtained by multiplying that for a single channel, while DC can be accounted for by modifying the value of $\lambda$. For radio channel we utilize the"log-distance path loss model" [8], similar to what was done in [7] and setting the very same parameters.

Also, we introduce the following two assumptions:

Assumption 1. All EDs are in the same radio conditions and if there is a simultaneous transfer from two or more EDs with the same $S F$, none of the packets is successfully received by the GW.

Assumption 2. If no other ED transmits with $S F_{k}$, the GW receives this packet with probability one. In the case if the transmissions of the two devices with $S F_{i}$ and $S F_{k}$ overlap in time and $i$ is less than $k$, then the packet sent with $S F_{i}$ is received.

\section{B. SF assignment optimization problem formulation}

Our goal is to maximize the correct packet delivery rate (PDR), under which we understand the ratio of the uplink packets correctly received by a LoRaWAN GW to all the uplink packets sent by all EDs, and which is given by:

$$
P D R=\sum_{i=k_{\min }}^{k_{\max }} \frac{n_{i} e^{-2 \lambda t_{i}}}{N}
$$

Here $n_{i}$ is the number of EDs operating using $S F_{i}$, and $N$ is the total number of EDs, $\lambda$ is the intensity of the data flow from the EDs, $t_{i}$ time of packet transfer while using $S F_{i}$ (see Table I), $k_{\min }$ and $k_{\max }$ are the minimum and maximum $S F$ possible, respectively. Specifically we are interested in such $n_{k_{\min }} \ldots n_{k_{\max }}$ that in the sum give $N$ and maximize the PDR.

\section{Optimization problem solution}

To solve this optimization problem, we use the method of Lagrange multipliers. First, we compose the Lagrange function

$$
\begin{gathered}
L\left(n_{k_{\min }}, n_{k_{\min }+1}, \ldots n_{k_{\max }}, \alpha\right)= \\
\sum_{i=k_{\min }}^{k_{\max }} \frac{n_{i} e^{-2 \lambda n_{i} t_{i}}}{N}+\alpha\left(N-\sum_{i=k_{\min }}^{k_{\max }} n_{i}\right),
\end{gathered}
$$

where $\alpha$ is the indefinite Lagrange multiplier.

Second, we compute the partial derivative of the function (2) by $n_{i}$.

$$
\frac{\partial L}{\partial n_{i}}=\frac{-\alpha N+e^{-2 \lambda t_{i} n_{i}}-2 \lambda t_{i} n_{i} e^{-2 \lambda t_{i} n_{i}}}{N},
$$

where $i=k_{\min }, \ldots, k_{\max }$. Next, we form the system of equations by equating to zero the partial derivatives of the Lagrange function.

$$
\frac{\partial L}{\partial n_{i}}=0,
$$

where $i=k_{\min }, \ldots, k_{\max }$. Thus we obtain a system of equations:

$$
e^{-2 \lambda t_{i} n_{i}}\left(1-2 \lambda t_{i} n_{i}\right)-\alpha N=0,
$$

where $i=k_{\min }, \ldots, k_{\max }$.

Since all equations of the system are the same and differ only in the value of the index $i$, the following equations must be satisfied for (5) to be true, which results in the following optimization problem:

$$
\left\{\begin{array}{c}
t_{k} n_{k}=t_{k_{\min }+1} n_{k_{\min }+1}=\ldots=t_{k_{\max }} n_{k_{\max }} \\
\sum_{i=k_{\min }}^{k_{\max }} n_{i}=N
\end{array},\right.
$$

where $i=k_{\min }, \ldots, k_{\max }$. Hence we obtain

$$
n_{i}=\frac{\frac{1}{t_{i}}}{\sum_{i=k_{\text {min }}}^{k_{\max }} \frac{1}{t_{i}}} \cdot N
$$


Conducting transformations and rounding the result to the nearest integer gives us the following solutions, defining the optimal number of EDs operating with $S F_{i}$ as

$$
n_{i}=\frac{\prod_{j=m_{\min }, j \neq i}^{k_{\max }} t_{j}}{\sum_{l=k_{\min }}^{k_{\max }} \prod_{j=k_{\min }, j \neq l}^{k_{\max }} t_{j}} \cdot N
$$

\section{Energy consumption}

In order to estimate the potential increase in the amount of the consumed by the EDs energy resulting from the use of proposed SF assignment strategy, we base on the results reported in [9]. Specifically, using equation (1) and the data from Table 5 from [9] we estimate the average current consumption of an ED operating with the different SFs as

$$
P_{\sum}=\sum_{i=k_{\min }}^{k_{\max }} I_{a v g_{S F_{i}}} V t_{S F_{i}}
$$

where $I_{a v g_{S F_{i}}}$ is the average current consumed (based on the data from [9]), $V$ is the supply voltage (3.5 $\mathrm{V}$ in our case), and $t_{S F_{i}}$ is the time for transmitting an uplink frame using SF $S F_{i}$ (see Table I).

\section{E. Illustrative example}

To illustrate the proposed SF assignment strategy let us consider the case when $N=1000$ located at the same distance (e.g., $1000 \mathrm{~m}$ ) from the $\mathrm{GW}$, and there are only two possible SFs: $S F 7$ and $S F 8$. In this case (1) can be written as

$$
P D R=\frac{n_{7} \cdot e^{-2 \lambda n_{i} t_{7}}}{n}+\frac{n_{8} \cdot e^{-2 \lambda n_{i} t_{8}}}{N}
$$

where $k_{\min }=7, k_{\max }=8$.

Fig. 1 illustrates the effect of the number of EDs utilizing $S F 8$ on the PDR for two illustrative values of $\lambda$. The $P D R$ is shown separately for the EDs operating with $S F 7$ (in red), $S F 8$ (in green), and for all $N$ EDs together (in blue). The number of EDs maximizing the $P D R$ for the network as whole, obtained with the proposed strategy, is marked with a black dot. Note, that the traditional method of SF assignment based exclusively on radio signal strength indicator (RSSI) for the described scenario would make all nodes transmit using the minimum SF possible, i.e., SF7. As one can see from Fig. 1 (number of EDs with SF8=0), the PDR in this case is about $20 \%$, while the proposed strategy increases it to over $33 \%$.

\section{EVALUATION}

To get a deeper insight into operation and utility of the proposed SF assignment method and to compare it against the SotA approaches, we conducted a set of simulations using NS-3 network simulator. The simulations were made using the LoRaWAN model developed by Van den Abeele et al. and utilized in [9], which is available from [10].

The logic of operation of the additional software implementing our proposed SF assignment strategy is as follows:

1) In the very beginning the SF is chosen based on PER 0.01 criterion [7].
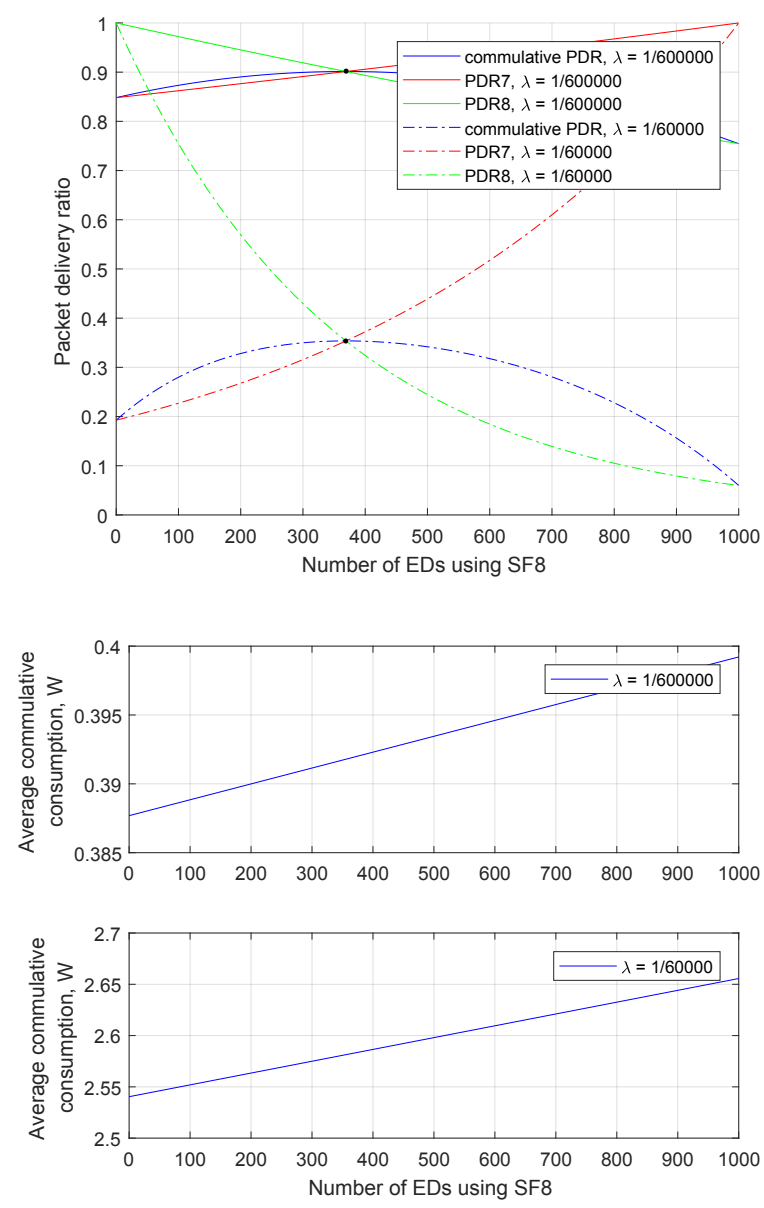

Fig. 1. Effect of the number of EDs on the $P D R$ for two single-SF cases (i.e., $S F 7$ and $S F 8$ ) and for the dual-SF network, with EDs distributed according to the proposed strategy.

2) Using equation (8) the number of EDs $n_{k_{\text {min }}}, n_{k_{\text {min }}+1}, \ldots, n_{k_{\max }} \quad$ using each $S F_{k_{\text {min }}}, S F_{k_{\text {min }}+1}, \ldots, S F_{k_{\text {max }}}$ is defined

3) The EDs are distributed between the SF groups, by randomly picking the necessary EDs for each group.

For our simulations we considered a network consisting of 100, 500, 1000, 5000 or 10000 class A LoRaWAN EDs located at a distance of $1 \mathrm{~km}$ from a single GW. All the other key parameters of our simulation match the ones already presented in Section II-A. The simulations were conducted for several scenarios, which differ in the number of the SF available. Namely, we considered the cases of a network utilizing two $\left(k_{\min }=7, k_{\max }=8\right)$, three $\left(k_{\min }=7, k_{\max }\right.$ $=9$ ) or six different SFs $\left(k_{\min }=7, k_{\max }=12\right)$. As a base-line scenario we also simulated the network utilizing the conventional LoRaWAN SF distribution strategy based on signal strength and PER 0.01 criterion ( [5] and [7]), which result in all nodes obtaining the minimum SF possible.

The results for the PDR, calculated as the ratio of the number of uplink packets received by the GW to that sent by the EDs, are depicted in Fig. 2. As one can see from the presented results, with the increase of the number of EDs in 


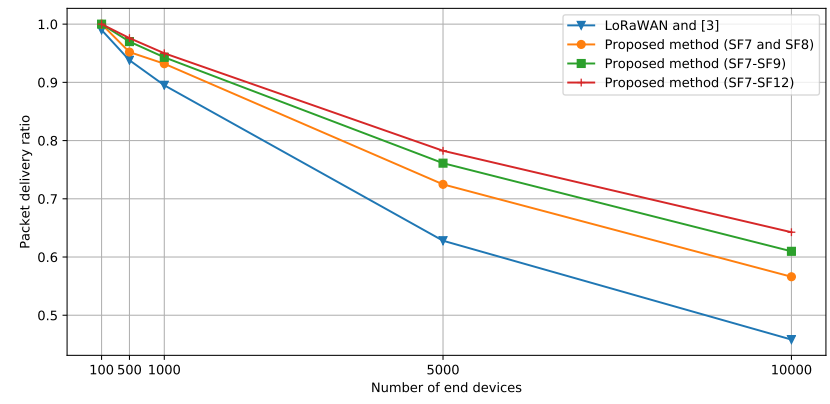

Fig. 2. Effect of the number of EDs on $P D R$ for different SF assignment strategies

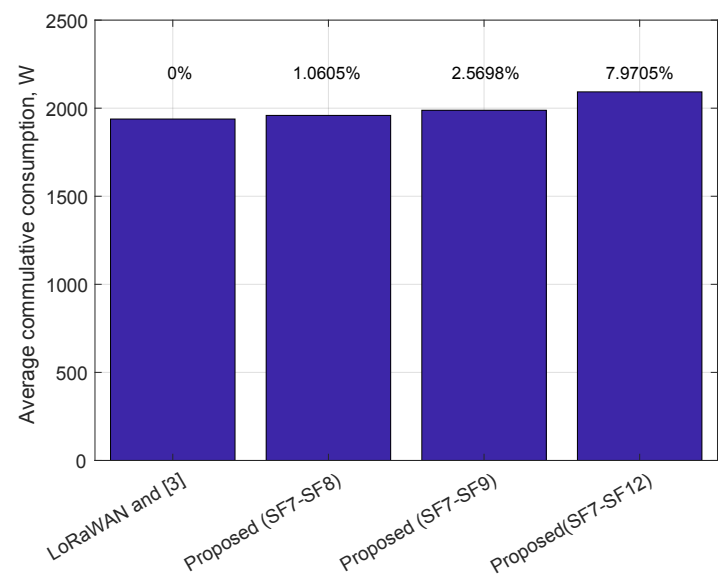

Fig. 3. Average network power consumption for different $S F$ assignment strategies for $10000 \mathrm{EDs}$

the network the PDR in the network operating following the proposed SF assignment strategy starts to outperform that of the SotA approaches. Specifically, for 5000 EDs the proposed strategy results in PDR increase of up to 22\%, and with 10000 EDs - up to $41 \%$ compared to the base line scenario. Also, as expected, having more SFs is more beneficial than having less.

To characterize the difference in the energy consumption between the proposed SF assignment strategy and the SotA one, we used the methodology described in Section II-D. The respective results and the average energy loss compared to the original approach are presented in Fig. 3. As one can see, use of two SFs and the proposed strategy results in the increase of the energy consumption by one percent, while for six SFs the average consumption is increased by eight percent. Note that, as this can be seen from Fig. 2, the former results in increase of PDR by $23 \%$, and the latter - by over $40 \%$ compared to that of the base line "min-time" strategy.

\section{CONCLUSION}

In the current paper we approached the problem of improving the scalability of a LoRaWAN LPWAN by enabling efficient selection of the SF parameter used by the end devices. We have formulated and solved the respective optimization problem, and demonstrated the operation of the proposed SF assignment strategy for a scenario when all end devices are located in identical radio conditions. The conducted simulations show that compared to the state-of-the-art approaches, suggested in [5] and [7], for dense networks the proposed method increases the probability of uplink data delivery by 20 to $40 \%$. The cost of it is the increase of the average power consumption by 1 to $8 \%$, respectively. Importantly, our results show that the state-of-the-art LoRaWAN SF assignment strategies are not optimal from the scalability point of view, and demonstrate one possible way how the scalability of LoRaWAN can be improved.

Note, that in this paper we have had quite some limitations. To start with, all end devices were located at the same distance from the base station. Next, we have not considered the presence of the capture effect, as well as the inter-SF interference. Also in this paper we have not addressed the possibility of transmit power control and the actual mechanisms and procedures for SF modification (e.g., the implementation of the adaptive data rate mechanism). Next, in our simulations we limited ourselves to the case when all the nodes are in identical radio conditions, transmit packets of the same size, and when there is just a single gateway. All these assumptions can be relaxed in further works. Also, the practical implementation and validation of the proposed SF assignment strategy "in field" can be of interest.

\section{ACKNOWLEDGMENT}

The work is carried out within the framework of the initiative scientific project No. 8.8540.2017 / 8.9 "Development of data transfer algorithms in IoT systems, taking into account restrictions on the complexity of devices". The work has also been financially supported by the Academy of Finland 6Genesis Flagship (grant 318927) and LPWAN evolution project funded by the University of Oulu.

\section{REFERENCES}

[1] S. Andreev, et al., "Understanding the iot connectivity landscape: a contemporary $\mathrm{m} 2 \mathrm{~m}$ radio technology roadmap," IEEE Commun. Mag., vol. 53, pp. 32-40, 2015.

[2] U. Raza, P. Kulkarni and M. Sooriyabandara, "Low power wide area networks: An overview,' IEEE Commun. Surv. \& Tut., vol. 19, no. 2, pp. 855-873, 2017.

[3] K. Mikhaylov, J. Petajajarvi and T. Hanninen, "Analysis of capacity and scalability of the lora low power wide area network technology," in Proc. Eur. Wireless Conf., 2016, pp. 1-6.

[4] LoRaWAN and Sigfox lead LPWANs, interoperability via compression, 2017. [Online]. Available: http://techblog.comsoc.org/2017/10/25/ lora-wan-and-sigfox-lead-lpwans-interoperability-via-compression/, [Accessed: 10-July-2018].

[5] N. Sornin et al., LoRaWAN specification 1.1, LoRa alliance, 2017.

[6] N. Sornin and A. Yegin, LoRaWAN 1.1 regional parameters, LoRa alliance, 2017.

[7] F. Van den Abeele, J. Haxhibeqiri, I. Moerman and J. Hoebeke, "Scalability analysis of large-scale lorawan networks in ns-3", IEEE Internet Things J., vol. 4, no. 6, pp. 2186-2198, 2017.

[8] ns3::logdistancepropagationlossmodel class reference, 2018. [Online]. Available: https://www.nsnam.org/doxygen/classns3_1_1_log_distance_ propagation_loss_model.html, [Accessed: 10-July-2018].

[9] L. Casals, B. Mir, R. Vidal and C. Gomez, "Modeling the energy performance of LoRaWAN", Sensors, vol. 17, no. 10, article 2364, 2017.

[10] imec-idlab/ns-3-dev-git, 2018. [Online]. Available: https://github.com/ imec-idlab/ns-3-dev-git/tree/lorawan, [Accessed: 10-July-2018]. 